Klaus Schlichte

\title{
Die Internationalen Beziehungen als Kirche, die Friedens- und Konfliktforschung als Sekte?
}

\author{
Anmerkungen zur Disziplin
}

In den vergangenen zehn Jahren hat die Friedens- und Konfliktforschung vor allem in Form neuer Studiengänge einen ungeahnten Aufschwung in Deutschland erlebt. Doch schon früher hat sich ein eher kritisches Segment der IB in der Friedens- und Konfliktforschung mit anderen Fächern verbunden. Ob daraus nun ein neues Fach entsteht, ist eine noch offene Frage. Für die IB entsteht aber nicht daraus eine veränderte Lage als Disziplin, sondern durch die Entdeckung des Globalen auch in anderen Sozialwissenschaften. Die Grenzen und der Kern der Disziplin werden unschärfer. Doch anstatt darauf mit »Disziplinierung"zu antworten, gilt es den Pluralismus des Faches zu stärken, um seine gesellschaftliche Anschlussfähigkeit und seine Attraktivität zu erhalten.

\section{Einleitung}

In welchem Verhältnis stehen Friedens- und Konfliktforschung und die Internationalen Beziehungen (IB) ${ }^{1}$ zueinander? Lässt sich ein thematischer Kern der »Friedens- und Konfliktforschung« identifizieren? Sollte diese eine dezidierte normative Ausrichtung haben und welche sollte das sein?

So fragen die beiden geschäftsführenden Herausgeber der »Zeitschrift für Internationale Beziehungen« (ZIB), aber der Anlass dieser Fragen ist nicht ganz klar. Vielleicht zeigen die Fragen Befindlichkeiten an, über die an dieser Stelle nur Mutmaßungen möglich sind. Eine Vermutung bestünde darin, dass sich innerhalb der Internationalen Beziehungen als Gemeinde eine Unsicherheit über das eigene Fach abzeichnet, die sich nun am Aufschwung der Friedens- und Konfliktforschung festmacht. Die zweite Vermutung besteht darin, dass der mehr gespürte als erforschte Hintergrund dieser Diskussion in realhistorischen Prozessen besteht, die auch eine Veränderung der universitären Fächer bewirken. Beide Vermutungen stehen hinter den folgenden Ausführungen, und weil es nur Vermutungen sind, ist die Irrtumswahrscheinlichkeit hoch.

Entsprechend läuft dieses Statement auf ein Plädoyer für Entspannung und Pluralismus hinaus: Die Kirche der IB muss sich keine Sorgen machen, weil es ein paar

1 Ich folge mit diesen Bezeichnungen den etablierten Formen in der Selbstbetrachtung in der ZIB. Das Kürzel »IB« bezeichnet demzufolge in erster Linie die in der DVPW-Sektion »Internationale Beziehungen« organisierte scientific community, die auch die »Zeitschrift für Internationale Beziehungen« unterhält. 
neue Studiengänge und eine neue Zeitschrift (die »Zeitschrift für Friedens- und Konfliktforschung«, kurz »ZeFKo«) gibt. Die Friedens- und Konfliktforschung ist keine Konkurrenz zu den ungleich stärker institutionalisierten »Internationalen Beziehungen«. Eine Disziplinierung ist nicht nötig. Grund über das eigene Schicksal nachzudenken hat die Fachgemeinde aber dennoch: Der Prozess der globalen Vergesellschaftung ist auch von anderen sozialwissenschaftlichen Disziplinen nicht unbemerkt geblieben, und schon jetzt hat »die IB« ihre vielleicht früher vorhandene Alleinstellung bei der Behandlung des Globalen verloren.

Der erste Teil dieses Statements wird sich dem Verhältnis zwischen Internationalen Beziehungen (IB) und Friedens- und Konfliktforschung widmen, der zweite den weiteren Fragen der Herausgeber dieses Forums, die auf Perspektiven der Forschung und die normativen Implikationen bzw. Verankerungen der Friedens- und Konfliktforschung zielen.

Dabei beziehen sich die folgenden Ausführungen mangels genauerer Kenntnis institutioneller und wissenschaftspolitischer Zusammenhänge im Ausland auf die Entwicklungen im deutschen Sprachraum. Die Verhältnisse zwischen akademischen Disziplinen sind natürlich nicht global identisch, weil auch ihre Inhalte nach meist sprachlich segmentierten Kulturräumen unterschiedlich sind, ebenso wie ihre politischen und gesellschaftlichen Rahmenbedingungen, die sich in Finanzierungen und Personalpolitik äußern.

Die gelegentlichen religionssoziologischen Parallelisierungen sollen das Gemeinte veranschaulichen und sind in gewissem Sinn ein parodistisches Stilmittel, aber eben nicht nur. Ihren Realgrund hat diese Parallele zum einen in der analogen Funktion der Wissenschaft als Orientierungsmittel oder symbolische Form, in der die Welt intellektuell zugänglich gemacht werden soll, und zum anderen in den analogen Hierarchien. Als Weltanschauung ist die moderne Wissenschaft eben das funktionale Äquivalent zum religiösen Weltbild. Und es ist soziologisch durchaus interessant zu sehen, dass sich viele kirchliche Sozialformen in der Wissenschaft erhalten haben, von der Predigt über die Priesterweihe bis zum Kirchentag, oder eben zum Hirtenbrief.

\section{Friedens- und Konfliktforschung - ein Fach oder nur ein Studiengang? Fluch oder Segen von Bologna?}

Das Kernthema der Friedens- und Konfliktforschung sind soziale Konflikte, ihre ursächlichen Prozesse, ihre inneren Dynamiken und ihre Folgen. Diese Forschung zu diesem Gegenstand speist sich aus unterschiedlichen fachlichen, theoretischen und methodischen Traditionen. Nimmt man die Mitgliedschaftsstruktur der »Arbeitsgemeinschaft Friedens- und Konfliktforschung« zur Grundlage, dann erkennt man darin zwar einen starken Anteil von Politikwissenschaftlern, besonders von Vertretern der IB. Aber reduzieren lassen sich weder die Themen noch die scientific community der Friedens- und Konfliktforschung auf diesen Anteil. Auch in anderen Ländern und in den meisten gleichnamigen Studiengängen ist erkennbar, 
dass sich hier mehrere Disziplinen treffen. Friedens- und Konfliktforschung wird in Bradford, Tromsø oder an der University of Notre Dame von Psychologen, Historikern, Geographen, Soziologen oder Pädagogen ebenso betrieben wie von Politikwissenschaftlern.

Friedens- und Konfliktforschung teilt diese auf einen Gegenstand konzentrierte, aber eigenartige Zwischenstellung mit einigen anderen Feldern, die ebenfalls interdisziplinär besetzt sind. Man könnte die Area Studies oder Regionalwissenschaften nennen, oder die sicherlich prominenteren Lebenswissenschaften. In beiden Feldern wird mit sehr unterschiedlichen disziplinären Hintergründen an ähnlichen oder teilweise identischen Gegenständen gearbeitet.

Dieses Zusammenspiel ist nicht einfach, aber es ist auch nicht notwendig problematisch. Schließlich schwanken in allen etablierten Wissenschaften die Fachidentitäten. In der Geographie ist nicht erkennbar, wie Klimatologie und politische Geographie genau zusammenhängen. In der Politikwissenschaft gibt es eine Politische Soziologie, in der Soziologie genauso. Mal ist in der Rechtswissenschaft die Rechtssoziologie zentral, dann wieder über Jahrzehnte gleichsam inexistent, kurz: So klar wie sie tun, sind die etablierten Fächer auch nicht. Weder sind ihre Gegenstände einheitlich definiert, noch sind ihre Methoden intern unumstritten oder singulär.

Warum ist die Friedens- und Konfliktforschung trotz ihrer notorischen Unschärfe, trotz ihres fragwürdigen Fachcharakters erfolgreich? Der Aufstieg der Friedensund Konfliktforschung in Deutschland hat viel mit wissenschaftspolitischen Vorgängen zu tun. Denn ein Gutteil der Differenzierungen in Fächer und akademische Felder jüngerer Zeit sind den wissenschaftspolitischen Einflüssen geschuldet, die allgemein als »Bologna« bezeichnet wird. Dieser unendliche Prozess der Reform universitärer Ausbildung hat im deutschen Sprachraum zu einer Vielzahl neuer Studiengänge geführt. Dadurch erhielt die als akademisches Fach nicht ausgeprägte Friedens- und Konfliktforschung neue Sichtbarkeit. In Marburg, Magdeburg, Frankfurt am Main, Hamburg, Augsburg, Tübingen und Konstanz entstanden neue Magisterstudiengänge, an denen überwiegend Politikwissenschaftler beteiligt sind, die das Fach, eng verbunden mit der Soziologie, der Philosophie, der Psychologie und der Geschichts- und Kulturwissenschaften, unterrichten. Die rege Nachfrage nach Studienplätzen in diesen Studiengängen und die angekündigte Neugründung der oben bereits angesprochenen »Zeitschrift für Friedens- und Konfliktforschung « für das Jahr 2012 dürften im Wesentlichen der Anlass für die Klärung des Verhältnisses sein. ${ }^{2}$

Die Verwirrung teilen aber nicht alle: Dadurch, dass Friedens- und Konfliktforschung je nach Standort ein etwas anderes Gepräge hat und sich die Gewichtungen

2 Die über die Jahre eigentlich erstaunlich rege Selbstbeobachtung, sowohl der IB wie auch der Friedens- und Konfliktforschung, hat die beiden Themen »Bologna« und »Exzellenzinitiative« bisher nicht reflektiert, obwohl diese die beiden nachhaltigsten wissenschaftspolitischen Einflüsse auf beide Fächer der letzten zwanzig Jahre gewesen sein dürften, vgl. Münch (2007), Maeße (2010) und zur Logik der konstanten Reform im europäischen Wissenschaftsraum Bruno (2008). 
der Fächer darin stark unterscheiden, ist ihre Konturierung als Fach zwar in Abgrenzung zu anderen sozialwissenschaftlichen Studiengängen nach wie vor unklar. Alle an den Auswahlverfahren für diese Studiengänge Beteiligten werden aber bemerkt haben, dass die Studierenden mit diesen Differenzen sehr kundig umgehen. Sie wissen, welches Profil das Fach an welchem Ort hat. An der Frage der methodischen Orientierung wird das besonders erkennbar. ${ }^{3}$ Diese Unterschiede können schwerlich durch übergeordnete Entscheidungen eingeebnet werden. Tatsächlich gibt es im deutschen Wissenschaftssystem auch gar keine Instanz, die über Fachbezeichnungen und -inhalte entscheiden könnte. Ein Vorteil des Föderalismus ist, dass er Differenz erlaubt.

Zugleich fällt es Studierenden oft schwer, sich selbständig zu orientieren und Schwerpunkte zu setzen. Dieses ZIB-Forum zeigt, dass auch andere Probleme haben, die Kerninhalte der Friedens- und Konfliktforschung von anderen Fächern zu unterscheiden. Denn Friedens- und Konfliktforschung kann weder auf eine etablierte Reihe von Einführungen verweisen, noch auf einen festen methodischen Kanon, der sich von anderen Disziplinen unterscheiden würde.

Der Vorteil dieser gelebten Interdisziplinarität ist aber die Breite der Möglichkeiten. Das Fach hat keinen mainstream, sondern nährt sich aus unterschiedlichen Auffassungen über seinen Gegenstand. Deshalb sind soziologisch wie geschichtswissenschaftlich verankerte Themen ebenso möglich wie genuin politikwissenschaftliche oder klassische Themen der IB zur Außenpolitik, zu zwischenstaatlichen Kriegen oder zu Rüstungsdynamiken. In Friedens- und Konfliktforschung kann man eben auch zu Repräsentationen von Fremdheit im deutsch-türkischen Rap forschen oder zur Soziologie russischer Führungsschichten oder zu Kommunikationsprozessen in der Mediation. Wie an den Standorten in Marburg, Magdeburg und Hamburg erkennbar wird, zeigen die an diesem Studiengang beteiligten Lehrenden im Unterschied zur IB eine größere Neigung, sich Methoden der Mikrosoziologie und der ethnologischen Feldforschung zu eigen $\mathrm{zu}$ machen und über »das Globale« nicht nur abstrakt zu diskutieren, sondern es in seinen konkreten Besonderheiten in Feldforschungen auch außerhalb Europas zu untersuchen (vgl. Schatz 2009; Schlichte 2012).

Aus dieser Beobachtung des Fachs Friedens- und Konfliktforschung als Studiengang kann man je nach Einstellung Unterschiedliches folgern: »Bologna« war segensreich oder eben ein Verhängnis. Die Interdisziplinarität, die die von »Bologna« begünstigte Friedens- und Konfliktforschung auszeichnet, ist ihr bisheriger großer Vorteil, weil dies eine viel größere thematische Breite ermöglicht. Diese Begegnung zwischen den Fächern im Rahmen neuer Studiengänge kann schließlich auch

3 Der wichtigste Unterschied scheint mir hier im Anteil kulturwissenschaftlicher Elemente in den Curricula und Studieninhalten zu sein. Je nach Studienort gibt es unterschiedliche Traditionen und unterschiedliches Personal und, im Falle von Friedens- und Konfliktforschung, auch unterschiedliche disziplinäre Kombinationen. Während das Fach zum Beispiel in Frankfurt am Main klar politikwissenschaftlich dominiert ist, wird in Magdeburg ein starker Anteil von Historikern, Philosophen und Anglisten bestritten. Zur Entwicklung des Fachs in der akademischen Lehre vgl. Brühl (2005), zur Anlage der verschiedenen Studiengänge in Deutschland vgl. Imbusch/Zoll (2005). 
für die Lehrenden hilfreich sein. So mühselig die Koordination eines fachübergreifenden Studiengangs sein mag, sie bietet den Lehrenden die Chance, in der Auseinandersetzung Methoden, Traditionen und Thematiken anderer Fächer intensiver kennenzulernen, als dies über Lehrbücher möglich ist. Das hilft dabei, die fachliche Ethnisierung der Wissenschaft zu vermeiden.

$\mathrm{Ob}$ sich durch die Entwicklung neuer Studiengänge dauerhaft neue wissenschaftliche Fachgliederungen ergeben, bleibt abzuwarten. Es wird letztlich davon abhängen, ob sich die bestehenden Disziplinen als flexibel genug erweisen, auf veränderte gesellschaftliche Wahrnehmungen und Bewertungen zu reagieren. Man sollte die Entwicklung einer neuen Generation von Konfliktforscherinnen und Konfliktforschern $^{4}$ aber nicht zur IB-Feindschaft hypostasieren. Sie folgt einem schon lange existierenden Muster der personellen Überlappung zwischen der Friedens- und Konfliktforschung und den Internationalen Beziehungen.

\section{Personalidentität-Priester zweier Kirchen?}

Mindestens in Deutschland, sicher aber auch in anderen Ländern, hat sich seit langem eine beträchtliche Überschneidung der Personenkreise ergeben, die in der Disziplin Internationale Beziehungen und in der Friedens- und Konfliktforschung lehren und forschen. Auch inhaltlich überschneiden sich die beiden akademischen Felder erheblich, denn die Frage nach Krieg und Frieden war doch die Gründungsfrage der Internationalen Beziehungen als akademische Disziplin, auch wenn man sie heute vielleicht eher in der Unterscheidung von Konflikt und Kooperation thematisiert. Die Überlappung gibt es aber bereits seit den 1970er Jahren. Die Generation von Ulrich Albrecht, Lothar Brock, Klaus Jürgen Gantzel, Ekkehart Krippendorf, Volker Rittberger und Dieter Senghaas praktizierte das gleiche straddling, das heute für Irritation sorgt. ${ }^{5}$ Es äußerte sich eben nur nicht in eigenen Studiengängen der Friedens- und Konfliktforschung, sondern seit 1968 in der Arbeit und den Publikationen der ebenfalls interdisziplinären Vereinigung »Arbeitsgemeinschaft Friedensund Konfliktforschung«. Dabei ist es interessant zu sehen, dass die gleichzeitige Existenz der beiden Fächer zugleich zu einer Art Sortierung führte: Während sich die kritischen Autoren eher in der Friedens- und Konfliktforschung sammelten, entwickelte sich jedenfalls die in der DVPW organisierte IB mehr und mehr zu einem Hort des funktionalistisch-liberalen Paradigmas. Gibt es also doch zwei Kirchen?

Die Persistenz der Friedens- und Konfliktforschung könnte man also als Reaktion auf dominante Paradigmen der DVPW-IB interpretieren. An einem Gründungsthema der IB, dem Krieg, wird das deutlich. Der Krieg war darin zwischen 1990

4 Zur Selbstbeschreibung dieser auch nicht mehr ganz jungen »jüngeren« Generation vgl. Fischer/Sahm (2005) oder auch das DFG-Netzwerk »Konstruktivistische Konfliktforschung «.

5 Vgl. die Beiträge in Senghaas (1972) sowie rückblickend auf die Geschichte der Friedensforschung in Deutschland Sahm et al. (2002), Wasmuth (1998) sowie die Beiträge von Brühl, Brzoska und Müller in diesem Heft. 
und 2005 fast ein Anathema, der Konflikt ohnehin eher eine Pathologie als eine produktive soziale Beziehung. Der Hypostasierung der »Zivilmacht Europa« zur normativen Avantgarde der Weltgeschichte stand in der deutschen DVPW-IB ein Bild der anderen Weltregionen gegenüber, das diese nur als rückständige und durch global governance zu zivilisierende Regionen mit begrenzter Staatlichkeit, defekten Demokratien und governance-Defiziten thematisieren konnte (vgl. Fach/Simonis 2000; Schlichte 2006). Nur die USA nehmen in diesem wirklich eurozentrischen Weltbild eine eigenartige Position ein. Es ist deshalb auch nicht verwunderlich, dass der für die transatlantischen Beziehungen und die europäische »Wertegemeinschaft« gleichermaßen peinliche Irak-Krieg in diesem Paradigma nicht zum Forschungsgegenstand wurde. Nicht die »Antinomien des demokratischen Friedens« (Geis et al. 2007) sind zur großen Avenue der Forschung geworden, sondern die innerstaatlichen Kriege, in deren Behandlung sich die gedachte Zweiteilung der Welt in »zones of peace« und »zones of turmoil« (Singer/Wildavsky 1993), in ein friedliches »Imperium« und Zonen der »Barbaren« (Rufin 1991) wiederholt. Im Unterschied zur Ethnologie, zu weiten Teilen der Soziologie und zur Geschichtswissenschaft ist diese mentale Teilung der Welt in den IB noch nicht überwunden worden. In der Friedens- und Konfliktforschung ist dagegen eine kritische Distanz zu dieser Weltsicht allgemeiner Standard. Entsprechend überwiegen in der dominanten IB auch heute affirmative Haltungen zur europäischen Politik, in der Friedens- und Konfliktforschung dagegen kritische. In der Friedens- und Konfliktforschung hat die These einer irgendwie gearteten Überlegenheit Europas die Konfrontation mit der Kritischen Theorie, dem Poststrukturalismus und den Postcolonial Studies nicht überlebt. ${ }^{6}$

Ein Blick auf die Kriegsforschung ist auch anregend für die zweite These dieses Beitrags, demzufolge sich eine Krise der IB abzeichnet. Ihr Kern und ihre Konturen sind unklarer geworden, sicherlich in Zusammenhang mit ihrem Wachstum, gemessen an der Zahl der Studierenden, großen Verbundforschungen, Graduiertenschulen und Drittmittelprojekten.

Eine drittmittelfinanzierte Forschung zu innerstaatlichen Kriegen gab es innerhalb der deutschen IB in der Mitte der 1990er Jahre nur an der Universität Hamburg (vgl. Gantzel/Siegelberg 1990) und an der Universität Heidelberg (Pfetsch 1991). Der innerstaatliche Krieg war sonst eher Angelegenheit der regionalwissenschaftlich arbeitenden Sozialwissenschaften. Im Jahr 2011 sieht die Situation ganz anders aus: Gegenwärtig wird von Vertreterinnen der IB dazu an der FU Berlin, Konstanz, Greifswald, Osnabrück, Frankfurt am Main, Marburg, Tübingen, Duisburg, Köln und Bremen in größerem Umfang geforscht. Diese Entwicklung hat ihre Gründe jedenfalls nicht im Gegenstand, denn die Zahl innerstaatlicher Kriege hat seit 1992 kontinuierlich abgenommen (AKUF 2012). Mutmaßlich sind veränderte politische Aufmerksamkeiten hier stärker ursächlich wirksam.

6 Empirisch nachweisbar gibt es eine analoge Differenz innerhalb der US-amerikanischen IB-Szene, vgl. Hamati-Ataya (2011). 
Zugleich hat diese erneute Beschäftigung mit dem Thema Krieg der IB in ihrer Krise nicht weitergeholfen. Denn die Untauglichkeit der Theorien des IB-Mainstream für die Erklärung oder auch nur Beschreibung der Kriegsdynamik nach 1945 war immer offensichtlich (vgl. Gantzel/Siegelberg 1990; Zangl/Zürn 2003). Doch die Forschungen zu Kriegen und bewaffneten Konflikten sind in den vergangenen zehn Jahren so genau und so kleinteilig geworden, dass der Bezug zu Fragen der internationalen Beziehungen kaum noch gewahrt wurde. Betrachtet man die jüngeren Publikationen aus diesen Forschungen, so wird häufig nicht klar, ob es sich nicht eher um Beiträge zur vergleichenden Politikwissenschaft (Schneckener 2002), zur politischen Soziologie (Schlichte 2009) oder Internationalen Politischen Ökonomie (Bussmann/Schneider 2007) handelt. Ein neuer theoretischer Impuls ist, gemessen an den zuvor bestehenden theoretischen Ansätzen, aus dieser Forschung für die IB nicht generiert worden. Zugleich wurden die Übergänge zu anderen Disziplinen fließender.

Die Herausforderung in diesem Forschungsfeld wird sicher darin bestehen, die Vielzahl der Erkenntnisse wieder mit Grundfragen der Internationalen Beziehungen zusammenzuführen. Welche Wirkung hat der Boom der Interventionspolitik der letzten zwanzig Jahre für die Architekturen von Macht und Herrschaft in der Weltgesellschaft? Der durchaus produktive Beitrag der Friedens- und Konfliktforschung kann dabei darin bestehen, nicht nur wie üblich internationale Institutionen, sondern die Internationalisierung von vermeintlich »nationalen« Formen des Regierens, aber auch die lokalen Internationalisierungen mit in den Blick zu nehmen.

\section{Eine Krise der IB?}

Die Frage nach dem Verhältnis zwischen den beiden akademischen Feldern könnte den Verdacht erwecken, es ginge hier um Versuche der Unter- bzw. Überordnung. Wird »die Disziplin« IB unruhig, weil ihr Konkurrenten erwachsen? Sollte sich, so fragen die Herausgeber in der Einladung zum ZIB-Symposium im Oktober 2011, Friedens- und Konfliktforschung nicht besser als Security Studies, also zu einem Teilgebiet der IB erklären?

Haben die Priester der IB Angst vor den Sekten? Derartige Versuche der Unterordnung wären natürlich wider den Sinn akademischer Tätigkeit. Dass die Wissenschaft sich in Disziplinen gliedert, sollte niemanden dazu veranlassen, Disziplinierungen vorzunehmen. Jenseits der natürlich auch nicht ganz klaren Vorstellungen darüber, wie sich Wissenschaft von Nicht-Wissenschaft unterscheidet, ist der Sinn solcher Debatten zweifelhaft. Die Friedens- und Konfliktforschung ist jedenfalls von der schieren Größe ihrer community und der für sie verwendeten Ressourcen keine Gefahr für die IB. Das Fach ist außerdem nicht institutionalisiert genug, um eine ernsthafte Konkurrenz für die viel etabliertere IB darzustellen.

Es scheint umgekehrt ebenso wenig sinnvoll, die Verwaltungsgrenzen der Wissenschaft zu Unterschieden in der Sache zu hypostasieren. Was als Fach Anerkennung findet und was nicht, das entscheidet sich im gesellschaftlichen Raum, nicht 
durch professoralen Konsens, der, wie wir seit Ludwik Fleck (1980) wissen, Innovationen nur aufhalten, aber nicht verhindern kann. Auch wie genau hier die Hierarchien verlaufen würden ist ganz unklar, denn es gäbe ebenso gute Gründe, IB und Politikwissenschaft nur Teil der »Menschenwissenschaften«, und insofern als Unterfälle von Soziologie oder Geschichtswissenschaft zu begreifen.

Die Diskussion über das Verhältnis zwischen den Fächern könnte aber auch auf eine Krise der IB verweisen. Ist nicht ihr eigener Gegenstand längst undeutlich geworden? Was ist heute der Kern der Disziplin? Das Programm der global governance scheint dafür jedenfalls nicht hinreichend $\mathrm{zu}$ sein, ebenso wenig wie das Paradigma der globalen Integration oder eine Zentrierung um die Frage der Demokratisierung.

Setzt man die Entwicklung der deutschsprachigen IB in Beziehung zu Entwicklungen in anderen Sozialwissenschaften, werden Konvergenzen - oder je nach Lesart auch Konkurrenzen - gegenwärtig vor allem in der Geschichtswissenschaft erkennbar. Die Innovationen, die mit viel Sorgfalt zur transnationalen Geschichte in den vergangenen zehn Jahren entstanden sind, ${ }^{7}$ sollten der IB vielleicht mehr Gedanken verursachen als ein paar neue MA-Programme. Die Zukunft könnte noch ganz andere »Gefahren« bergen: Was wäre, wenn die Soziologie eines Tages entdecken sollte, dass es auch außerhalb Deutschlands und sogar außerhalb Europas Gesellschaft gibt? Bisher hat auch die Soziologie das Globale nur allgemein thematisiert. Die Forschung über soziale Realitäten außerhalb der OECD ist jedoch für sie bis heute nur ein Thema der »Entwicklungssoziologie«.

Sorgen müssen sich aber nur solche Vertreter des Faches machen, denen die Fachidentität wichtiger ist als die Auseinandersetzung über die Sache, die theoretisch informierte und empirisch gehaltvolle Forschung über politische Dynamiken der Weltgesellschaft. Teilt man die Sorgen über die Einheit der Kirche und die Konkurrenz der Konfessionen nicht, dann ist alles Neue willkommene Bereicherung des Denkens.

\section{Ein Plädoyer für Pluralismus}

Fachgliederungen haben keine Gründe im Gegenstand (vgl. Elias 1983). Sie entstanden einmal aus Denktraditionen, heute aber sind sie oft lediglich Organisationsprinzip - als administrative Gliederung nach bürokratischen Prinzipien der Universitäten in Forschung und Lehre. Dieses System wird von der Konkurrenz um Mittel und Stellen unter den solchermaßen einmal entstandenen Disziplinen ebenso stabilisiert wie von den Wissenschaftlerinnen und Wissenschaftlern, auf die Organisationsmacht einen besonderen Reiz ausübt und die deshalb gern zu Priestern, Bischöfen und Kardinälen ihrer Kirchen werden. Aus diesen beiden Quellen, der bürokratischen Ordnungsneigung und der Lust an der Organisation, droht dem produktiven

7 Für einen Überblick vgl. z.B. Loth/Osterhammel (2000); Conrad et al. (2007) und Büschel/ Speich (2009). 
Pluralismus wissenschaftlicher Fächer immer wieder Gefahr. Um diese höhere Produktivität des Pluralismus zu wahren, muss man sich nur diesen alten Widerspruch zwischen bürokratischer Organisation und demokratischer Praxis bewusst halten.

Der Pluralismus hat natürlich den Preis, dass die spezifischen Inhalte der IB an Trennschärfe verloren haben. Das hat seine Gründe aber auch in realen Entwicklungen, auf die andere Fächer ebenso reagieren und auf die durch neue fachliche Orientierungen reagiert wird, wie sich das eben im Aufschwung der Friedens- und Konfliktforschung erkennen lässt. Die nationalen Paradigmen verblassen, und auch die Zentralität Europas wird immer weniger plausibel. Die Globalisierung erreicht die Sozialwissenschaften gerade erst, was sich sowohl in der Internationalisierung der Themen wie ihrer Organisation und vielleicht auch einmal in Deutschland in der Internationalisierung ihres Personals ausdrücken wird.

Solange gerade dieser letzte Schritt nicht erreicht sein wird, muss der Pluralismus des Fachs, der international eine Selbstverständlichkeit ist, in Deutschland auf andere Weise erhalten werden. Drei Regeln könnten ihn befördern: Erstens reicht es, wenn Themen anknüpfungsfähig an mainstream-Diskussionen sind, sie müssen sich nicht in dessen Mitte bewegen. Zweitens sollte die Autonomie junger Forscherinnen und Forscher befördert werden, statt sie in Verbundprojekte mit vorgegebenen Thematiken zu zwängen, und drittens sollte die Forschung näher »zu den Sachen selbst« gehen, anstatt sich bloß aus Sekundäranalysen von Daten staatlicher und quasi-staatlicher Organisationen und amtlichen Publikationen zu nähren. ${ }^{8}$ Die Kultur der Quellenkritik in der Geschichtswissenschaft wie die Methodik der ethnologischen Feldforschung als »geplante Irritation« könnten hier anregende Vorbilder sein.

\section{Schluss: Brauchen wir eine normativ geleitete Friedens- und Konfliktfor- schung?}

Die Herausgeber haben schließlich die Frage gestellt, ob die Friedens- und Konfliktforschung ein besonderes normatives Fundament bräuchte. Nun ist es ein wissenschaftlicher Gemeinplatz, dass jede Forschung schon durch die Wahl ihrer Themen unvermeidlich normativen Gehalt hat (Weber 1988: 500). In der Friedens- und Konfliktforschung ist ebenso wenig wie in irgendeinem anderen Fach eine normative Orientierung dekretierbar, die nicht gesellschaftlich schon vorproduziert wäre. Und in der Tat lässt sich die frühere Friedens- und Konfliktforschung als eine Vereinigung von normativ einigermaßen Gleichgesinnten auffassen.

Heute ist dieser Konsens sicher geringer und lässt sich vielleicht als die Hoffnung definieren, dass physische Gewalt als Mittel der Politik überwunden werden möge. Ein größerer Konsens scheint nicht mehr definierbar, weil der ehemals strikt anti-

8 Das ist das alte Credo der Phänomenologie (vgl. Husserl 1980: 6). Zu ihrer Relevanz in den IB vgl. das Forum der Zeitschrift »International Political Sociology« $(2011,5: 1)$, zu ethnologischer Methoden für die Politikwissenschaft vgl. Schatz (2009). 
militaristische und kritische Konsens der westdeutschen Friedensforschung den Abschwung der Friedensbewegung und die neue Legitimierung der »humanitären Intervention« nicht überlebt hat (vgl. Fischer/Sahm 2005). Die mittlere und jüngere Generation in der Friedens- und Konfliktforschung ist normativ weniger eindeutig zurechenbar.

Wenn diese Eindrücke sich bei näherem Hinsehen bestätigen sollten, dann könnte meiner Ansicht nach statt »Friedens- und Konfliktforschung« auch die Selbstbezeichnung »Konfliktforschung« reichen. Denn der Begriff des Friedens hat als neuzeitlicher »Erwartungsbegriff« (Koselleck 2006: 68) vielleicht zu viele Konnotationen. Er weckt zudem die Erwartung, dass eine konfliktfreie Gesellschaft erstrebenswert sei. Die Betonung des Friedens verdeckt zu sehr die produktiven Seiten des Konflikts, wie dies auch in weiten Teilen der Terminologie zum Ausdruck kommt. Conflict prevention und conflict settlement etwa sind Ausdrücke, die der soziologischen Grundwahrheit entgegenstehen, dass Konflikte nicht nur ubiquitär sind, sondern der wesentliche Modus gesellschaftlichen und politischen Wandels.

Der große Verdienst der Politikwissenschaft, selbst in ihrer nur auf Institutionen reduzierten Form, ist ja die Einsicht, dass Institutionen den Austrag von Konflikten ermöglichen, sie versachlichen und so sozialen und politischen Wandel ohne tiefe Traumatisierungen möglich machen. Das könnte als normatives Programm reichen, befriedigt aber nicht das Bedürfnis nach »Heilsgütern und Offenbarungen spendenden Gnadengaben« (Weber 1988: 609), das alle Forschenden, Lehrenden und Studierenden immer wieder ergreift. Auch dieses moralische Bedürfnis, verbunden mit dem Bedürfnis nach Gemeinschaft, drängt die Wissenschaft in die Richtung religiöser Verbände.

Eine andere, durchaus konsequenzenreiche normative Frage stellt sich noch in Bezug auf das Verhältnis von Forschung und Regierung: Sollen die IB und die Friedens- und Konfliktforschung sich an den Bedürfnissen staatlicher Politik ausrichten? Ein einfaches Anschmiegen an die Außenpolitik selbst demokratischer Staaten kann sicher nicht mit dem Verweis auf demokratische Willensbildung begründet werden (vgl. Meyer 2012), denn einerseits ist empirisch die Deckung zwischen der Außenpolitik von Regierungen und Mehrheitsmeinungen häufig nicht gegeben, wie am aktuellen Fall des Krieges in Afghanistan nur besonders deutlich wird. Andererseits entspricht eine sozialwissenschaftliche Forschung, die bloße Verlängerung von Regierungspolitik ist, nicht dem Verständnis demokratischer Wissenschaft. Professoren werden in Deutschland durch Schwüre auf das Volk und die Verfassung, nicht auf Regierungen oder Minister vereidigt. Eine praktische Konklusion dieser realen normativen Verankerung sollte aus meiner Sicht sein, dass sowohl die IB wie die Konfliktforschung ihre Ergebnisse einer breiteren Öffentlichkeit zugänglich machen und nicht nur in englischsprachigen, für den Laien unzugänglichen Zeitschriften publizieren. Zu dieser gesellschaftlichen Rückbindung gehört auch die Suche nach Vermittlung in die medialen und städtischen Öffentlichkeiten - ein Feld, in dem die deutschsprachige Konfliktforschung nach meinem Eindruck mehr Potential hat, als gegenwärtig nach außen sichtbar wird. 


\section{Literatur}

AKUF 2012: Webseite der Arbeitsgemeinschaft Kriegsursachenforschung, in: http://www.aku f.de; 17.03.2012.

Brzoska, Michael 2012: Friedensforschung und Internationale Beziehungen - Lob der Verschiedenheit, in: Zeitschrift für Internationale Beziehungen 19: 1, 127-141.

Bruno, Isabelle 2008: A vos marques, prêts... cherchez! La stratégie européenne de Lisbonne, vers un marché de la recherche, Brüssel.

Brühl, Tanja 2005: Die Friedensforschung an den Hochschulen. Auf dem Weg zum etablierten Studienfach?, in: Fischer, Sabine/Jahn, Egbert/Sahm, Astrid (Hrsg.): Die Zukunft des Friedens. Die Friedens- und Konfliktforschung aus der Perspektive der jüngeren Generation, Wiesbaden, 537-554.

Brühl, Tanja 2012: Friedensforschung als »Superwissenschaft« oder Sub-Disziplin? Zum Verhältnis der Friedens- und Konfliktforschung und der Internationalen Beziehungen, in: Zeitschrift für Internationale Beziehungen 19: 1, 171-183.

Büschel, Hubertus/Speich, Daniel (Hrsg.) 2009: Entwicklungswelten. Globalgeschichte der Entwicklungszusammenarbeit, Frankfurt a. M.

Bussmann, Margit/Schneider, Gerald 2007: When Globalization Discontent Turns Violent: Foreign Economic Liberalization and Internal War, in: International Studies Quarterly 51: 1, 79-97.

Conrad, Sebastian/Eckert, Andreas/Freitag, Ulrike (Hrsg.) 2007: Globalgeschichte. Theorien, Ansätze, Themen, Frankfurt a. M.

Elias, Norbert 1983: Über den Rückzug der Soziologen auf die Gegenwart, in: Kölner Zeitschrift für Soziologie und Sozialpsychologie 35: 1, 29-40.

Fach, Wolfgang/Simonis Georg 2000: Die Welt des Autors. Eine Polemik, in: Zeitschrift für Internationale Beziehungen, 7: 2, 385-398.

Fischer, Sabine/Sahm, Astrid 2005: Friedensforschung und Normativität. Positionen der jüngeren Generation, in: Fischer et al. (Hrsg.): Die Zukunft des Friedens. Die Friedens- und Konfliktforschung aus der Perspektive der jüngeren Generation, Wiesbaden, 49-72.

Fleck, Ludwik 1980: Entstehung und Entwicklung einer wissenschaftlichen Tatsache. Einführung in die Lehre vom Denkstil und Denkkollektiv, Frankfurt a. M.

Gantzel, Klaus Jürgen/Siegelberg, Jens 1990: Krieg und Entwicklung. Überlegungen zur Theoretisierung von Kriegsursachen unter besonderer Berücksichtigung der Zeit seit 1945, in: Volker Rittberger (Hrsg.): Theorien der Internationalen Beziehungen. Bestandsaufnahme und Forschungsergebnisse, PVS-Sonderheft 21, Opladen, 219-239.

Geis, Anna/Müller, Harald/Brock, Lothar 2007: Demokratische Kriege als Antinomien des demokratischen Friedens. Eine komplementäre Forschungsagenda, in: Geis, Anna (Hrsg.): Schattenseiten des demokratischen Friedens. Zur Kritik einer Theorie liberaler Außen- und Sicherheitspolitik, Frankfurt a. M., 69-91.

Hamati-Ataya, Inanna 2011: Contemporary »Dissidence« in American IR: The New Structure of Anti-Mainstream Scholarship?, in: International Studies Perspectives 12: 4, 362-398.

Husserl, Edmund 1980: Logische Untersuchungen, Bd. II, Untersuchungen zur Phänomenologie und Theorie der Erkenntnis, 5. Auflage, Tübingen.

Imbusch, Peter/Zoll, Ralf (Hrsg.) 2005: Friedens- und Konfliktforschung. Eine Einführung, Wiesbaden.

Koselleck, Reinhart 2006: Begriffsgeschichten. Studien zur Semantik und Pragmatik der politischen und sozialen Sprache, Frankfurt a. M.

Loth, Wilfried/Osterhammel, Jürgen (Hrsg.) 2000: Internationale Geschichte. Themen - Ergebnisse - Aussichten, München.

Maeße, Jens 2010: Die vielen Stimmen des Bologna-Prozesses. Zur diskursiven Logik eine bildungspolitischen Programms, Bielefeld. 
Meyer, Christoph O. 2012: Normative, theoretische und praxeologische Defizite der Friedensforschung am Beispiel der Konfliktprävention, in: Zeitschrift für Internationale Beziehungen 19: 1, 185-197.

Münch, Richard 2007: Die akademische Elite, Frankfurt a. M.

Müller, Harald 2012: Über allen Gipfeln ist Ruh: Zum Verhältnis von Friedensforschung und IB, in: Zeitschrift für Internationale Beziehungen 19: 1, 155-169.

Pfetsch, Frank R. 1991: Konflikte seit 1945. Daten - Fakten - Hintergründe, Würzburg.

Rufin, Jean-Christophe 1991: L'empire et les nouveaux barbares, Paris.

Sahm, Astrid/Sapper, Manfred/Weichsel, Volker (Hrsg.) 2002: Die Zukunft des Friedens, Bd. I, Eine Bilanz der Friedens- und Konfliktforschung, Wiesbaden.

Schatz, Edward (Hrsg.) 2009: Political Ethnography: What Immersion Contributes to The Study of Power, Chicago, IL.

Schlichte, Klaus 2006: Ist Kritik noch möglich? Zum Verhältnis von Politik und Wissenschaft in den Internationalen Beziehungen, in: Gunther Hellmann (Hrsg.): Forschung und Beratung in der Wissensgesellschaft. Das Feld der internationalen Beziehungen und der Außenpolitik, Baden-Baden, 283-306.

Schlichte, Klaus 2009: In the Shadow of Violence: The Politics of Armed Groups, Frankfurt a. M.

Schlichte, Klaus 2012: Der Streit der Legitimitäten. Der Konflikt als Grund einer historischen Soziologie des Politischen, in: Zeitschrift für Friedens- und Konfliktforschung 1:1, i.E.

Schneckener, Ulrich 2002: Auswege aus dem Bürgerkrieg. Modelle zur Regulierung ethnonationalistischer Konflikte in Europa, Frankfurt a. M.

Senghaas, Dieter 1972: Friedensforschung und Gesellschaftskritik, Frankfurt a. M.

Singer, Max/Wildavsky, Aaron 1993: The Real World Order: Zones of Peace, Zones of Turmoil, Chatham, NJ.

Wasmuth, Ulrike 1998: Geschichte der deutschen Friedensforschung, Münster.

Weber, Max 1988: Der Sinn der Wertfreiheit der soziologischen und ökonomischen Wissenschaften, in: Gesammelte Aufsätze zur Wissenschaftslehre, 5. Auflage, Tübingen, 489-540.

Weber, Max 1988: Wissenschaft als Beruf, in: Gesammelte Aufsätze zur Wissenschaftslehre, 5. Auflage, Tübingen, 582-613.

Zangl, Bernhard/Zürn, Michael 2004: Frieden und Krieg, Frankfurt a. M. 